\title{
Effects of Non-Uniform Temperature Gradients on Triple Diffusive Marangoni Convection in a Composite Layer
}

\author{
Narayanappa Manjunatha', Ramakrishna Sumithra ${ }^{2}$ \\ ${ }^{1}$ School of Applied Sciences, REVA University, Bengaluru, India \\ ${ }^{2}$ Department of Mathematics, Government Science College, Bengaluru, India \\ Email: manjunatha.n@reva.edu.in,sumitra_diya@yahoo.com
}

How to cite this paper: Manjunatha, N. and Sumithra, R. (2019) Effects of NonUniform Temperature Gradients on Triple Diffusive Marangoni Convection in a Composite Layer. Open Journal of Applied Sciences, 9, 640-660.

https://doi.org/10.4236/ojapps.2019.98052

Received: July 23, 2019

Accepted: August 19, 2019

Published: August 22, 2019

Copyright () 2019 by author(s) and Scientific Research Publishing Inc. This work is licensed under the Creative Commons Attribution International License (CC BY 4.0).

http://creativecommons.org/licenses/by/4.0/

\begin{abstract}
The problem of triple diffusive Marangoni convection is investigated in a composite layer comprising an incompressible three component fluid saturated, sparsely packed porous layer over which lies a layer of the same fluid. The lower rigid surface of the porous layer and the upper free surface are considered to be insulating to temperature, insulating to both salute concentration perturbations. At the upper free surface, the surface tension effects depending on temperature and salinities are considered. At the interface, the normal and tangential components of velocity, heat and heat flux, mass and mass flux are assumed to be continuous. The resulting eigenvalue problem is solved exactly for linear, parabolic and inverted parabolic temperature profiles and analytical expressions of the thermal Marangoni number are obtained. The effects of variation of different physical parameters on the thermal Marangoni numbers for the profiles are compared.
\end{abstract}

\section{Keywords}

Triple Diffusive, Thermal Marangoni Convection, Composite Layer, Temperature Profiles

\section{Introduction}

There are many fluid systems containing more than two components occurring in nature and engineering applications. The subject of systems having multi components in porous and fluid layers has attracted many researchers due to its importance in the study of crystal growth, geothermally heated lakes, earth core, solidification of molten alloys, underground water flow, acid rain effects, natural 
phenomena such as contaminant transport, warming of stratosphere and magmas and their laboratory models and sea water etc. The effect of multicomponent convection is studied by Griffiths [1] [2], Rudraiah and Vortmeyer [3], Shivakumara [4], Poulikakos [5], Pearlstein et al. [6] and Lopez et al. [7].

For the fluid layer, Chand [8] has applied the linear stability analysis and a normal mode analysis to study the triple-diffusive convection in a micropolar ferromagnetic fluid layer heated and soluted from below. Suresh Chand [9] has investigated the triple-diffusive convection in a micropolar ferrofluid layer heated and soluted below subjected to a transverse uniform magnetic field in the presence of uniform vertical rotation. Shivakumara and Naveen Kumar [10] have investigated the effect of couple stresses on linear and weakly nonlinear stability of a triply diffusive fluid layer using a modified perturbation technique. Kango et al. [11] have studied the theoretical investigation of the triple-diffusive convection in a micropolar ferrofluid layer heated and soluted below subjected to a transverse uniform magnetic field in the presence of uniform vertical rotation. Vivek Kumar and Mukesh Kumar Awasthi [12] have considered the problem of triple-diffusive convection in a horizontal nanofluid layer heated and salted from below using linear stability theory and normal mode technique. A linear stability analysis is carried out for triple diffusive convection in Oldroyd-B liquid and rotating couple stress liquid by Sameena Tarannum and Pranesh [13] [14].

In porous medium, Suresh Chand [15] has obtained closed-form of solution for the rotation in a magnetized ferrofluid with internal angular momentum, heated and soluted from below saturating a porous medium and subjected to a transverse uniform magnetic field. Salvatore Rionero [16] have studied a triple convective-diffusive fluid mixture saturating a porous horizontal layer, heated from below and salted from above and below. Kango et al. [17] have studied the triple-diffusive convection in Walters (Model B') fluid with varying gravity field is considered in the presence of uniform vertical magnetic field in porous medium. Khan et al. [18] investigated the steady triple diffusive boundary layer free convection flow past a horizontal flat plate embedded in a porous medium filled by a water-based nanofluid and two salts. Moli Zhao et al. [19] have investigated the linear stability of triply diffusive convection in a binary Maxwell fluid saturated porous layer using modified Darcy-Maxwell model. The triply diffusive convection in a Maxwell viscoelastic fluid is mathematically investigated in the presence of uniform vertical magnetic field through porous medium studied by Pawan Kumar Sharma et al. [20] using linearized stability theory and normal mode analysis. Jyoti Prakash et al. [21] [22] have studied the magnetohydrodynamic triply diffusive convection with one of the components as heat, with diffusivity and sparsely distributed porous medium using the Darcy-Brinkman model. Rana et al. [23] have studied the triple-diffusive convection in a horizontal layer of nanofluid heated from below and salted from above and below. Goyal et al. [24] have studied the triple diffusive natural convection under Darcy flow 
over an inclined plate embedded in a porous medium saturated with a binary base fluid containing nanoparticles and two salts using group theory transformations. Patil et al. [25] studied a numerical investigation on steady triple diffusive mixed convection boundary layer flow past a vertical plate moving parallel to the free stream in the upward direction. A linear stability analysis is performed for the onset of triple-diffusive convection in the presence of internal heat source in a Maxwell fluid saturated porous layer studied by Mukesh Kumar Awasthi et al. [26]. Raghunatha et al. [27] have investigated the weakly nonlinear stability of the triple diffusive convection in a Maxwell fluid saturated porous layer. For the composite layers, Sumithra [28] has studied the triple-diffusive Marangoni convection in a two layer system and obtained the analytical expression for the Thermal Marangoni Number. The combined effects of magnetic field and non uniform basic temperature gradients on two component convection in two layer system is investigated by Manjunatha and Sumithra [29] [30].

This paper investigates the triple diffusive Marangoni convection in a composite layer and studies the effects of the linear, parabolic and inverted parabolic temperature gradients on the corresponding thermal Marangoni numbers.

\section{Mathematical Formulation}

Consider a three different diffusing components with different molecular diffusivities, saturating a horizontally isotropic sparsely packed porous layer of thickness $d_{m}$ underlying a three component fluid layer of thickness $d$. The lower surface of the porous layer is considered to be rigid and the upper surface of the fluid layer is free at which the surface tension effects depending on temperature and both the species concentrations. Both the boundaries are kept at different constant temperatures and salinities. A Cartesian coordinate system is chosen with the origin at the interface between porous and fluid layers and the $z$-axis, vertically upwards. The basic equations for fluid and porous layer respectively as

$$
\begin{gathered}
\nabla \cdot \boldsymbol{q}=0 \\
\rho_{0}\left[\frac{\partial \boldsymbol{q}}{\partial t}+(\boldsymbol{q} \cdot \nabla)\right]=-\nabla P+\mu \nabla^{2} \boldsymbol{q} \\
\frac{\partial T}{\partial t}+(\boldsymbol{q} \cdot \nabla) T=\kappa \nabla^{2} T \\
\frac{\partial C_{1}}{\partial t}+(\boldsymbol{q} \cdot \nabla) C_{1}=\kappa_{1} \nabla^{2} C_{1} \\
\frac{\partial C_{2}}{\partial t}+(\boldsymbol{q} \cdot \nabla) C_{2}=\kappa_{2} \nabla^{2} C_{2} \\
\nabla_{m} \cdot \boldsymbol{q}_{m}=0 \\
\rho_{0}\left[\frac{1}{\varepsilon} \frac{\partial \boldsymbol{q}_{m}}{\partial t}+\frac{1}{\varepsilon^{2}}\left(\boldsymbol{q}_{m} \cdot \nabla_{m}\right) \boldsymbol{q}_{m}\right]=-\nabla_{m} P_{m}+\mu_{m} \nabla_{m}^{2} \boldsymbol{q}_{m}-\frac{\mu}{K} \boldsymbol{q}_{m}
\end{gathered}
$$




$$
\begin{gathered}
A \frac{\partial T_{m}}{\partial t}+\left(\boldsymbol{q}_{m} \cdot \nabla_{m}\right) T_{m}=\kappa_{m} \nabla_{m}^{2} T_{m} \\
\varepsilon \frac{\partial C_{m 1}}{\partial t}+\left(\boldsymbol{q}_{m} \cdot \nabla_{m}\right) C_{m 1}=\kappa_{m 1} \nabla_{m}^{2} C_{m 1} \\
\varepsilon \frac{\partial C_{m 2}}{\partial t}+\left(\boldsymbol{q}_{m} \cdot \nabla_{m}\right) C_{m 2}=\kappa_{m 2} \nabla_{m}^{2} C_{m 2}
\end{gathered}
$$

Here $\boldsymbol{q}=(u, v, w)$ is the velocity vector, $\rho_{0}$ is the fluid density, $t$ is the time, $\mu$ is the fluid viscosity, $P$ is the pressure for fluid layer, $T$ is the temperature, $\kappa$ is the thermal diffusivity of the fluid, $\kappa_{1}$ and $\kappa_{2}$ is the solute 1 and solute 2 diffusivity of the fluid in the fluid layer, $C_{1}$ is the concentration 1 or the salinity field 1 for the fluid, $C_{2}$ is the concentration 2 or the salinity field 2 for the fluid in the fluid layer, $P_{m}$ is the pressure for porous layer, $K$ is the permeability of the porous medium, $A=\frac{\left(\rho_{0} C_{p}\right)_{m}}{\left(\rho C_{p}\right)_{f}}$ is the ratio of heat capacities, $C_{p}$ is the specific heat, $\varepsilon$ is the porosity, $\kappa_{m 1}$ and $\kappa_{m 2}$ is the solute1 and solute2 diffusivity of the fluid in porous layer, $C_{m 1}, C_{m 2}$ are the concentration 1 and concentration 2 for porous layer respectively and the subscripts " $m$ " and " $P$ " refer to the porous medium and the fluid respectively.

The Equations (1) to (10) have a basic steady solution for fluid and porous layer respectively.

$$
\begin{gathered}
\boldsymbol{q}=\boldsymbol{q}_{b}, P=P_{b}(z), T=T_{b}(z), C_{1}=C_{1 b}(z), C_{2}=C_{2 b}(z) \\
\boldsymbol{q}_{m}=\boldsymbol{q}_{m b}, P_{m}=P_{m b}\left(z_{m}\right), T_{m}=T_{m b}\left(z_{m}\right), C_{1 m}=C_{1 m b}\left(z_{m}\right), C_{2 m}=C_{2 m b}\left(z_{m}\right) \\
-\frac{\partial T_{b}}{\partial z}=\frac{T_{0}-T_{u}}{d} h(z) \text { in } 0 \leq z \leq d \\
-\frac{\partial T_{m b}}{\partial z_{m}}=\frac{T_{1}-T_{0}}{d_{m}} h_{m}\left(z_{m}\right) \text { in }-d_{m} \leq z_{m} \leq 0 \\
C_{1 b}(z)=C_{10}-\frac{\left(C_{10}-C_{1 u}\right) z}{d} \text { in } 0 \leq z \leq d \\
C_{1 m b}\left(z_{m}\right)=C_{10}-\frac{\left(C_{1 l}-C_{10}\right) z_{m}}{d_{m}} \text { in }-d_{m} \leq z_{m} \leq 0 \\
C_{2 b}(z)=C_{20}-\frac{\left(C_{20}-C_{2 u}\right) z}{d} \text { in } 0 \leq z \leq d \\
C_{2 m b}\left(z_{m}\right)=C_{20}-\frac{\left(C_{2 l}-C_{20}\right) z_{m}}{d_{m}} \text { in }-d_{m} \leq z_{m} \leq 0
\end{gathered}
$$

where $T_{0}=\frac{\kappa d_{m} T_{u}+\kappa_{m} d T_{l}}{\kappa d_{m}+\kappa_{m} d}, C_{10}=\frac{\kappa_{1} d_{m} C_{1 u}+\kappa_{1 m} d C_{1 l}}{\kappa_{1} d_{m}+\kappa_{1 m} d}, C_{20}=\frac{\kappa_{2} d_{m} C_{2 u}+\kappa_{2 m} d C_{2 l}}{\kappa_{2} d_{m}+\kappa_{2 m} d}$ are the interface temperature and concentrations, $h(z)$ and $h_{m}\left(z_{m}\right)$ are temperature gradients in fluid and porous layers respectively and the subscript "b" denotes the basic state.

To examine the stability of the system, we give a small perturbation to the 
system as

$$
\begin{aligned}
\boldsymbol{q}=\boldsymbol{q}_{b}+\boldsymbol{q}^{\prime}, P & =P_{b}+P^{\prime}, T=T_{b}(z)+\theta, C_{1}=C_{1 b}(z)+S_{1}, C_{2}=C_{2 b}(z)+S_{2} \\
\boldsymbol{q}_{m}= & \boldsymbol{q}_{m b}+\boldsymbol{q}_{m}^{\prime}, P_{m}=P_{m b}+P_{m}^{\prime}, T_{m}=T_{m b}\left(z_{m}\right)+\theta_{m}, \\
C_{1 m}= & C_{1 m b}\left(z_{m}\right)+S_{m 1}, C_{2 m}=C_{2 m b}\left(z_{m}\right)+S_{m 2}
\end{aligned}
$$

where the primed quantities are the dimensionless one. Introducing (19) \& (20) are substituted into the (1) to (10), apply curl twice to eliminate the pressure term from (2) to (7) and only the vertical component is retained. The variables are then nondimensionalised using $\frac{d^{2}}{\kappa}, \frac{\kappa}{d}, T_{0}-T_{u}, C_{10}-C_{1 u}, C_{20}-C_{2 u}$ in the fluid layer and $\frac{d_{m}^{2}}{\kappa_{m}}, \frac{\kappa_{m}}{d_{m}}, T_{l}-T_{0}, C_{1 l}-C_{10}, C_{2 l}-C_{20}$ as the corresponding characteristic quantities in the porous layer.

To render the equations nondimensional, we choose different scales for the two layers (Chen and Chen [31], Nield [32]), so that both layers are of unit length such that $(x, y, z)=d\left(x^{\prime}, y^{\prime}, z^{\prime}\right),\left(x_{m}, y_{m}, z_{m}\right)=d_{m}\left(x_{m}^{\prime}, y_{m}^{\prime}, z_{m}^{\prime}-1\right)$.

Omitting the primes for simplicity, we get in $0 \leq z \leq 1$ and $0 \leq z_{m} \leq 1$ respectively

$$
\begin{gathered}
\frac{1}{\operatorname{Pr}} \frac{\partial}{\partial t}\left(\nabla^{2} W\right)=\nabla^{4} W \\
\frac{\partial \theta}{\partial t}=W h(z)+\nabla^{2} \theta \\
\frac{\partial S_{1}}{\partial t}=W+\tau_{1} \nabla^{2} S_{1} \\
\frac{\partial S_{2}}{\partial t}=W+\tau_{2} \nabla^{2} S_{2} \\
\operatorname{Pr}_{m} \frac{\partial}{\partial t}\left(\nabla_{m}^{2} W_{m}\right)=\hat{\mu} \beta^{2} \nabla_{m}^{4} W_{m}-\nabla_{m}^{2} W_{m} \\
A \frac{\partial \theta_{m}}{\partial t}=W_{m} h_{m}\left(z_{m}\right)+\nabla_{m}^{2} \theta_{m} \\
\varepsilon \frac{\partial S_{m 1}}{\partial t}=W_{m}+\tau_{m 1} \nabla_{m}^{2} S_{m 1} \\
\varepsilon \frac{\partial S_{m 2}}{\partial t}=W_{m}+\tau_{m 2} \nabla_{m}^{2} S_{m 2}
\end{gathered}
$$

Here, for the fluid layer, $\operatorname{Pr}=\frac{v}{\kappa}$ is the Prandtl number, $\tau_{1}=\frac{\kappa_{1}}{\kappa}$ is the ratio of salute1 diffusivity to thermal diffusivity fluid in fluid layer, $\tau_{2}=\frac{\kappa_{2}}{\kappa}$ is the ratio of salute2 diffusivity to thermal diffusivity fluid in fluid layer. For the porous layer, $P r_{m}=\frac{\varepsilon v_{m}}{\kappa_{m}}$ is the Prandtl number, $\beta^{2}=\frac{K}{d_{m}^{2}}=D a$ is the Darcy number, $\beta$ is the porous parameter, $\hat{\mu}=\frac{\mu_{m}}{\mu}$ is the viscosity ratio, $\tau_{m 1}=\frac{\kappa_{m 1}}{\kappa_{m}}$ is the 
ratio of salute1 diffusivity to thermal diffusivity of the porous layer, $\tau_{m 2}=\frac{\kappa_{m 2}}{\kappa_{m}}$ is the ratio of salute2 diffusivity to thermal diffusivity of the porous layer, $h(z)$ and $h_{m}\left(z_{m}\right)$ are the non-dimensional temperature gradients with $\int_{0}^{1} h(z) \mathrm{d} z=1$ and $\int_{0}^{1} h_{m}\left(z_{m}\right) \mathrm{d} z_{m}=1, \theta$ and $\theta_{m}$ are the temperature in fluid and porous layers respectively, $S$ and $S_{m}$ are the concentration in fluid and porous layers respectively and $W$ and $W_{m}$ are the dimensionless vertical velocity in fluid and porous layer respectively.

We apply normal mode expansion on dependent variables as follows:

$$
\begin{gathered}
{\left[\begin{array}{c}
W \\
\theta \\
S_{1} \\
S_{2}
\end{array}\right]=\left[\begin{array}{c}
W(z) \\
\theta(z) \\
S_{1}(z) \\
S_{2}(z)
\end{array}\right] f(x, y) \mathrm{e}^{n t}} \\
{\left[\begin{array}{c}
W_{m} \\
\theta_{m} \\
S_{m 1} \\
S_{m 2}
\end{array}\right]=\left[\begin{array}{c}
W_{m}\left(z_{m}\right) \\
\theta_{m}\left(z_{m}\right) \\
S_{m 1}\left(z_{m}\right) \\
S_{m 2}\left(z_{m}\right)
\end{array}\right] f_{m}\left(x_{m}, y_{m}\right) \mathrm{e}^{n_{m} t}}
\end{gathered}
$$

with $\nabla_{2}^{2} f+a^{2} f=0$ and $\nabla_{2 m}^{2} f_{m}+a_{m}^{2} f_{m}=0$. Here $a$ and $a_{m}$ are the nondimensional horizontal wave numbers, $n$ and $n_{m}$ are the frequencies. Since the dimensional horizontal wave numbers must be the same for the fluid and porous layers, we must have $\frac{a}{d}=\frac{a_{m}}{d_{m}}$ and hence $a_{m}=\hat{d} a$.

Introducing Equation (29) and Equation (30) into the Equations (21) to (28) and denoting $\frac{\partial}{\partial z}=D$ and $\frac{\partial}{\partial z_{m}}=D_{m}$ then we get an eigenvalue problem consisting of the following ordinary differential equations in $0 \leq z \leq 1$ and $0 \leq z_{m} \leq 1$ respectively

$$
\begin{gathered}
\left(D^{2}-a^{2}+\frac{n}{P r}\right)\left(D^{2}-a^{2}\right) W=0 \\
\left(D^{2}-a^{2}+n\right) \theta+W h(z)=0 \\
\left(\tau_{1}\left(D^{2}-a^{2}\right)+n\right) S_{1}+W=0 \\
\left(\tau_{2}\left(D^{2}-a^{2}\right)+n\right) S_{2}+W=0 \\
{\left[\left(D_{m}^{2}-a_{m}^{2}\right) \hat{\mu} \beta^{2}+\frac{n_{m} \beta^{2}}{P r_{m}}-1\right]\left(D_{m}^{2}-a_{m}^{2}\right) W_{m}=0} \\
\left(D_{m}^{2}-a_{m}^{2}+A n_{m}\right) \theta_{m}+W_{m} h_{m}\left(z_{m}\right)=0 \\
\left(\tau_{m 1}\left(D_{m}^{2}-a_{m}^{2}\right)+n_{m} \varepsilon\right) S_{m 1}+W_{m}=0
\end{gathered}
$$




$$
\left(\tau_{m 2}\left(D_{m}^{2}-a_{m}^{2}\right)+n_{m} \varepsilon\right) S_{m 2}+W_{m}=0
$$

It is known that the principle of exchange of instabilities holds for triple diffusive convection in both fluid and porous layers separately for certain choice of parameters. Therefore, we assume that the principle of exchange of instabilities holds even for the composite layers. In other words, it is assumed that the onset of convection is in the form of steady convection and accordingly we take $n=n_{m}=0$. We get, in $0 \leq z \leq 1$ and $0 \leq z_{m} \leq 1$ respectively

$$
\begin{gathered}
\left(D^{2}-a^{2}\right)^{2} W=0 \\
\left(D^{2}-a^{2}\right) \theta+W h(z)=0 \\
\tau_{1}\left(D^{2}-a^{2}\right) S_{1}+W=0 \\
\tau_{2}\left(D^{2}-a^{2}\right) S_{2}+W=0 \\
{\left[\left(D_{m}^{2}-a_{m}^{2}\right) \hat{\mu} \beta^{2}-1\right]\left(D_{m}^{2}-a_{m}^{2}\right) W_{m}=0} \\
\left(D_{m}^{2}-a_{m}^{2}\right) \theta_{m}+W_{m} h_{m}\left(z_{m}\right)=0 \\
\tau_{m 1}\left(D_{m}^{2}-a_{m}^{2}\right) S_{m 1}+W_{m}=0 \\
\tau_{m 2}\left(D_{m}^{2}-a_{m}^{2}\right) S_{m 2}+W_{m}=0
\end{gathered}
$$

\section{Boundary Conditions}

The boundary conditions are nondimensionlised then subjected to normal mode analysis and finally they take the form

$$
\begin{gathered}
D^{2} W(1)+M a^{2} \theta(1)+M_{s 1} a^{2} S_{1}(1)+M_{s 2} a^{2} S_{2}(1)=0, \\
W(1)=D \theta(1)=D S_{1}(1)=D S_{2}(1)=0, \\
W_{m}(0)=D W_{m}(0)=D_{m} \theta_{m}(0)=D_{m} S_{m 1}(0)=D_{m} S_{m 2}(0)=0, \\
\hat{T} W(0)=W_{m}(1), \hat{T} \hat{d} D W(0)=D_{m} W_{m}(1), \\
\hat{T} \hat{d}^{2}\left(D^{2}+a^{2}\right) W(0)=\hat{\mu}\left(D_{m}^{2}+a_{m}^{2}\right) W_{m}(1), \\
\hat{T} \hat{d}^{3} \beta^{2}\left(D^{3} W(0)-3 a^{2} D W(0)\right)=D_{m} W_{m}(1)+\hat{\mu} \beta^{2}\left(D_{m}^{3} W_{m}(1)-3 a_{m}^{2} D_{m} W_{m}(1)\right), \\
\theta(0)=\hat{T} \theta_{m}(1), D \theta(0)=D_{m} \theta_{m}(1), S_{1}(0)=\widehat{S_{1}} S_{m 1}(1), \\
D S_{1}(0)=D_{m} S_{m 1}(1), S_{2}(0)=\widehat{S_{2}} S_{m 2}(1), D S_{2}(0)=D_{m} S_{m 2}(1)
\end{gathered}
$$

where $\widehat{S_{1}}=\frac{\kappa_{s 1}}{\kappa_{s 1 m}}, \widehat{S_{2}}=\frac{\kappa_{s 2}}{\kappa_{s 2 m}}$ are the ratios of solute1 and solute2 diffusivities of fluid layer to those of porous layer respectively, $\hat{d}=\frac{d_{m}}{d}=$ depth ratio, $\hat{T}=\frac{\kappa}{\kappa_{m}}=$ ratio of thermal diffusivities of fluid, $M=-\frac{\partial \sigma_{t}}{\partial T} \frac{\left(T_{0}-T_{u}\right) d}{\mu \kappa}$ is the Thermal Marangoni number, $M_{s 1}=-\frac{\partial \sigma_{t}}{\partial C} \frac{\left(C_{10}-C_{1 u}\right) d}{\mu \kappa}$ is the solute1 Maran- 
goni number and $M_{\mathrm{s} 2}=-\frac{\partial \sigma_{t}}{\partial C} \frac{\left(C_{20}-C_{2 u}\right) d}{\mu \kappa}$ is the solute2 Marangoni number.

\section{Method of Solution}

From Equation (39) and Equation (43), we get $W$ and $W_{m}$ as

$$
\begin{gathered}
W(z)=A_{1} \cosh a z+A_{2} z \cosh a z+A_{3} \sinh a z+A_{4} z \sinh a z \\
W_{m}\left(z_{m}\right)=A_{5} \cosh a_{m} z_{m}+A_{6} \sinh a_{m} z_{m}+A_{7} \cosh \delta_{m} z_{m}+A_{8} \sinh \delta_{m} z_{m}
\end{gathered}
$$

where $\delta_{m}=\sqrt{\frac{a_{m}^{2}+1}{\hat{\mu} \beta^{2}}}$ and $A_{i}^{\prime} s(i=1,2, \cdots, 8)$ are arbitrary constants, $W(z)$ and $W_{m}\left(z_{m}\right)$ are suitably written as

$$
\begin{gathered}
W(z)=A_{1}\left[\cosh a z+a_{1} z \cosh a z+a_{2} \sinh a z+a_{3} z \sinh a z\right] \\
W_{m}\left(z_{m}\right)=A_{1}\left[a_{4} \cosh a_{m} z_{m}+a_{5} \sinh a_{m} z_{m}+a_{6} \cosh \delta_{m} z_{m}+a_{7} \sinh \delta_{m} z_{m}\right]
\end{gathered}
$$

where

$$
\begin{aligned}
& a_{1}=\frac{a_{6} \Delta_{12}+a_{7} \Delta_{13}}{\hat{T} \hat{d}}, a_{2}=\frac{a_{6} \Delta_{10}+a_{7} \Delta_{11}}{\Delta_{9}}, a_{3}=\frac{a_{6} \Delta_{5}+a_{7} \Delta_{6}-\Delta_{8}}{\Delta_{7}}, \\
& a_{4}=-a_{6}, a_{5}=\frac{-a_{7} \delta_{m}}{a_{m}}, a_{6}=\Delta_{19}, a_{7}=\frac{\Delta_{17}}{\Delta_{18}} \\
& \Delta_{1}=\cosh \delta_{m}-\cosh a_{m}, \Delta_{2}=\sinh \delta_{m}-\frac{\delta_{m} \sinh a_{m}}{a_{m}}, \\
& \Delta_{3}=\delta_{m} \sinh \delta_{m}-a_{m} \sinh a_{m}, \Delta_{4}=\delta_{m}\left(\cosh \delta_{m}-\cosh a_{m}\right) \text {, } \\
& \Delta_{5}=\left(\delta_{m}^{2}+a_{m}^{2}\right) \cosh \delta_{m}-2 a_{m}^{2} \cosh a_{m}, \\
& \Delta_{6}=\left(\delta_{m}^{2}+a_{m}^{2}\right) \sinh \delta_{m}-2 a_{m} \delta_{m} \sinh a_{m}, \\
& \Delta_{7}=\frac{2 a \hat{T} \hat{d}^{2}}{\hat{\mu}}, \Delta_{8}=\frac{2 a^{2} \hat{T} \hat{d}^{2}}{\hat{\mu}}, \Delta_{9}=-2 a^{3} \hat{T} \hat{d}^{3} \beta^{2}, \\
& \Delta_{10}=a_{m} \sinh a_{m}+2 a_{m}^{2} \hat{\mu} \beta^{2} \sinh a_{m}-\delta_{m} \sinh \delta_{m}+\Delta_{100}, \\
& \Delta_{100}=\hat{\mu} \beta^{2}\left(\delta_{m}^{3} \sinh \delta_{m}-3 a_{m}^{2} \delta_{m} \sinh \delta_{m}\right), \\
& \Delta_{11}=a_{m} \cosh a_{m}+2 a_{m}^{2} \hat{\mu} \beta^{2} \cosh a_{m}-\delta_{m} \cosh \delta_{m}+\Delta_{110}, \\
& \Delta_{110}=\hat{\mu} \beta^{2}\left(\delta_{m}^{3} \cosh \delta_{m}-3 a_{m}^{2} \delta_{m} \cosh \delta_{m}\right), \\
& \Delta_{12}=\Delta_{3}-a \frac{\Delta_{10}}{\Delta_{9}}, \Delta_{13}=\Delta_{4}-a \frac{\Delta_{11}}{\Delta_{9}}, \\
& \Delta_{14}=\frac{\Delta_{12} \cosh a}{\hat{T} \hat{d}}+\left(\frac{\Delta_{10} \Delta_{7}+\Delta_{5} \Delta_{9}}{\Delta_{7} \Delta_{9}}\right) \sinh a, \\
& \Delta_{15}=\frac{\Delta_{13} \cosh a}{\hat{T} \hat{d}}+\left(\frac{\Delta_{11} \Delta_{7}+\Delta_{6} \Delta_{9}}{\Delta_{7} \Delta_{9}}\right) \sinh a, \\
& \Delta_{16}=\frac{\Delta_{8} \sinh a}{\Delta_{7}}-\cosh a, \Delta_{17}=\hat{T} \Delta_{14}-\Delta_{1} \Delta_{16} \text {, }
\end{aligned}
$$




$$
\Delta_{18}=\Delta_{2} \Delta_{14}-\Delta_{1} \Delta_{15}, \Delta_{19}=\frac{1}{\Delta_{1}}\left(\hat{T}-\frac{\Delta_{2} \Delta_{17}}{\Delta_{18}}\right) .
$$

We get the species concentration for fluid layer $S_{1}, S_{2}$ from (41) and (42) also from (45) and (46) species concentration for porous layer $S_{m 1}, S_{m 2}$ as

$$
\begin{gathered}
S_{1}(z)=A_{1}\left[a_{12} \cosh a z+a_{13} \sinh a z+\frac{f(z)}{\tau_{1}}\right] \\
S_{2}(z)=A_{1}\left[a_{16} \cosh a z+a_{17} \sinh a z+\frac{f(z)}{\tau_{2}}\right] \\
S_{m 1}\left(z_{m}\right)=A_{1}\left[a_{14} \cosh a_{m} z_{m}+a_{15} \sinh a_{m} z_{m}+\frac{f_{m}\left(z_{m}\right)}{\tau_{m 1}}\right] \\
S_{m 2}\left(z_{m}\right)=A_{1}\left[a_{18} \cosh a_{m} z_{m}+a_{19} \sinh a_{m} z_{m}+\frac{f_{m}\left(z_{m}\right)}{\tau_{m 2}}\right]
\end{gathered}
$$

where

$$
\begin{aligned}
& f(z)=R_{1}-R_{2}, f_{m}\left(z_{m}\right)=-\left(R_{3}+R_{4}\right), \\
& R_{1}=\frac{z}{4 a^{2}}\left[\left(a_{1}-a z a_{3}\right) \cosh a z+\left(a_{3}-a z a_{1}\right) \sinh a z\right], \\
& R_{2}=\frac{Z}{2 a}\left(\sinh a z+a_{2} \cosh a z\right), \\
& R_{3}=\frac{1}{\delta_{m}^{2}-a_{m}^{2}}\left(a_{6} \cosh \delta_{m} z_{m}+a_{7} \sinh \delta_{m} z_{m}\right), \\
& R_{4}=\frac{z_{m}}{2 a_{m}}\left(a_{4} \sinh a_{m} z_{m}+a_{5} \cosh a_{m} z_{m}\right), \\
& a_{12}=\widehat{S_{1}}\left(a_{14} \cosh a_{m}+a_{15} \sinh a_{m}\right)-\Delta_{27}, \\
& a_{13}=\frac{1}{a}\left(a_{m} a_{15} \cosh a_{m}+a_{14} a_{m} \sinh a_{m}+\Delta_{28}\right), \\
& a_{14}=\frac{\Delta_{30}}{\Delta_{31}}, a_{15}=\frac{\Delta_{29}}{a_{m}}, a_{16}=\widehat{S_{2}}\left(a_{18} \cosh a_{m}+a_{19} \sinh a_{m}\right)-\Delta_{32}, \\
& a_{17}=\frac{1}{a}\left(a_{18} a_{m} \sinh a_{m}+a_{19} a_{m} \cosh a_{m}+\Delta_{33}\right), \\
& a_{18}=\frac{\Delta_{36}}{\Delta_{37}}, a_{19}=\frac{\Delta_{35}}{a_{m}}, \Delta_{26}=\frac{1}{\tau_{1}}\left[\Delta_{22}\right], \Delta_{27}=\frac{\Delta_{20} \widehat{S_{1}}}{\hat{T} \tau_{m 1}}, \\
& \Delta_{28}=\frac{1}{\tau_{1}}\left(\frac{2 a a_{2}-a_{1}}{4 a^{2}}\right)-\frac{1}{\tau_{m 1}}\left[\frac{1}{2 a_{m}}\left(a_{4} \sinh a_{m}+a_{5} \cosh a_{m}\right)+\Delta_{280}\right] \text {, } \\
& \Delta_{280}=\frac{1}{2}\left(a_{5} \sinh a_{m}+a_{4} \cosh a_{m}\right)+\frac{\delta_{m}}{\delta_{m}^{2}-a_{m}^{2}}\left(a_{6} \sinh \delta_{m}+a_{7} \cosh \delta_{m}\right), \\
& \Delta_{29}=\frac{1}{\tau_{m 1}}\left(\frac{a_{5}}{2 a_{m}}+\frac{a_{7} \delta_{m}}{\delta_{m}^{2}-a_{m}^{2}}\right),
\end{aligned}
$$




$$
\begin{gathered}
\Delta_{30}=\Delta_{26}-\Delta_{28} \cosh a+a \Delta_{27} \sinh a-\Delta_{29} \cosh a \cosh a_{m}+\Delta_{300}, \\
\Delta_{300}=-\frac{\Delta_{29}}{a_{m}}\left(\widehat{S_{1}} a \sinh a \sinh a_{m}\right), \\
\Delta_{31}=\widehat{S_{1}} a \sinh a \cosh a_{m}+a_{m} \cosh a \sinh a_{m}, \Delta_{32}=\frac{\tau_{m 1} \widehat{S_{2} \Delta_{27}}}{\tau_{m 2} \widehat{S_{1}}} \\
\Delta_{33}=\frac{1}{\tau_{2}}\left(\frac{2 a a_{2}-a_{1}}{4 a^{2}}\right)-\frac{1}{\tau_{m 2}}\left[\frac{1}{2 a_{m}}\left(a_{4} \sinh a_{m}+a_{5} \cosh a_{m}\right)+\Delta_{280}\right], \\
\Delta_{34}=\frac{1}{\Delta_{22} \tau_{2}}\left[\Delta_{26} \tau_{1}\right], \Delta_{35}=\frac{1}{\tau_{m 2}}\left(\frac{a_{5}}{2 a_{m}}+\frac{a_{7} \delta_{m}}{\delta_{m}^{2}-a_{m}^{2}}\right), \\
\Delta_{36}=\Delta_{34}-\Delta_{33} \cosh a+a \Delta_{32} \sinh a-\Delta_{35} \cosh a \cosh a_{m}-\Delta_{360}, \\
\Delta_{360}=-\frac{\Delta_{35}}{a_{m}}\left(\widehat{S_{2}} a \sinh a \sinh a_{m}\right), \\
\Delta_{37}=\widehat{S_{2}} a \sinh a \cosh a_{m}+a_{m} \cosh a \sinh a_{m}
\end{gathered}
$$

\subsection{Linear Temperature Profile}

For this case

$$
h(z)=h_{m}\left(z_{m}\right)=1
$$

Substituting Equation (56) into the heat Equation (40) and Equation (44), we get $\theta$ and $\theta_{m}$ as

$$
\begin{gathered}
\theta(z)=A_{1}\left[a_{8} \cosh a z+a_{9} \sinh a z+f(z)\right] \\
\theta_{m}\left(z_{m}\right)=A_{1}\left[a_{10} \cosh a_{m} z_{m}+a_{11} \sinh a_{m} z_{m}+f_{m}\left(z_{m}\right)\right]
\end{gathered}
$$

where

$$
\begin{gathered}
a_{8}=\hat{T}\left(a_{10} \cosh a_{m}+a_{11} \sinh a_{m}\right)-\Delta_{20}, \\
a_{9}=\frac{1}{a}\left(a_{10} a_{m} \sinh a_{m}+a_{11} a_{m} \cosh a_{m}-\Delta_{21}\right), \\
a_{10}=\frac{\Delta_{24}}{\Delta_{25}}, a_{11}=\frac{\Delta_{23}}{a_{m}}, \\
\Delta_{20}=\frac{\hat{T}}{2 a_{m}}\left(a_{4} \sinh a_{m}+a_{5} \cosh a_{m}\right)+\frac{\hat{T}}{\delta_{m}^{2}-a_{m}^{2}}\left(a_{7} \sinh \delta_{m}+a_{6} \cosh \delta_{m}\right), \\
\Delta_{21}=-\left(\frac{2 a a_{2}-a_{1}}{4 a^{2}}\right)+\frac{1}{2 a_{m}}\left(a_{4} \sinh a_{m}+a_{5} \cosh a_{m}\right)+\Delta_{330}, \\
\Delta_{22}=\frac{1}{4 a^{2}}\left[\left(\left(a^{2}-1\right) a_{3}+2 a\right) \sinh a+\left(\left(a^{2}-1\right) a_{1}+2 a a_{2}\right) \cosh a\right]+\Delta_{220}, \\
\Delta_{220}=\frac{1}{4 a}\left[\left(a_{1}+2 a a_{2}\right) \sinh a+\left(a_{3}+2 a\right) \cosh a\right], \\
\Delta_{23}=\frac{a_{5}}{2 a_{m}}+\frac{a_{7} \delta_{m}}{\delta_{m}^{2}-a_{m}^{2}},
\end{gathered}
$$




$$
\begin{gathered}
\Delta_{24}=\Delta_{22}+\Delta_{21} \cosh a+a \Delta_{20} \sinh a+\Delta_{23} \cosh a \cosh a_{m}-\Delta_{240}, \\
\Delta_{240}=\frac{\Delta_{23}}{a_{m}}\left(\hat{T} a \sinh a \sinh a_{m}\right), \\
\Delta_{25}=\hat{T} a \sinh a \cosh a_{m}+a_{m} \cosh a \sinh a_{m} .
\end{gathered}
$$

The Thermal Marangoni number for this model obtained from $(47)^{1}$ and is found to be

$$
M_{1}=-\frac{\Lambda_{1}+\Lambda_{2}+\Lambda_{3}}{\Lambda_{4}}
$$

where

$$
\begin{gathered}
\Lambda_{1}=\left(a^{2}+a^{2} a_{1}+2 a a_{3}\right) \cosh a+\left(a^{2} a_{3}+a^{2} a_{2}+2 a a_{1}\right) \sinh a, \\
\Lambda_{2}=M_{s 1} a^{2}\left[a_{12} \cosh a+a_{13} \sinh a-\frac{\Omega_{1}}{\tau_{1}}\right], \\
\Lambda_{3}=M_{s 2} a^{2}\left[a_{16} \cosh a+a_{17} \sinh a-\frac{\Omega_{1}}{\tau_{2}}\right], \\
\Lambda_{4}=a^{2}\left[a_{8} \cosh a+a_{9} \sinh a-\Omega_{1}\right], \\
\Omega_{1}=\frac{1}{4 a^{2}}\left[\left(2 a+a a_{1}-a_{3}\right) \sinh a+\left(2 a a_{2}-a_{1}+a a_{3}\right) \cosh a\right]
\end{gathered}
$$

\subsection{Parabolic Temperature Profile}

We consider the profile as following (Sparrow et al. [33]):

$$
h(z)=2 z \text { and } h_{m}\left(z_{m}\right)=2 z_{m}
$$

Substituting Equation (60) into the heat Equation (40) and Equation (44), we get $\theta$ and $\theta_{m}$ as

$$
\begin{gathered}
\theta(z)=A_{1}\left[a_{20} \cosh a z+a_{21} \sinh a z+L(z)\right] \\
\theta_{m}\left(z_{m}\right)=A_{1}\left[a_{22} \cosh a_{m} z_{m}+a_{23} \sinh a_{m} z_{m}+L_{m}\left(z_{m}\right)\right]
\end{gathered}
$$

where

$$
\begin{gathered}
L(z)=-\left(R_{5}+R_{6}\right), L_{m}\left(z_{m}\right)=-\left(R_{7}+R_{8}\right), \\
R_{5}=\left(\frac{z^{2}}{2 a}-\frac{a_{2} z}{2 a^{2}}\right) \sinh a z+\left(\frac{a_{2} z^{2}}{2 a}-\frac{z}{2 a^{2}}\right) \cosh a z, \\
R_{6}=\left(\frac{\left(2 a^{2} z^{3}+3 z\right) a_{1}-3 a a_{3} z^{2}}{6 a^{3}}\right) \sinh a z+\left(\frac{\left(2 a^{2} z^{3}+3 z\right) a_{3}-3 a a_{1} z^{2}}{6 a^{3}}\right) \cosh a z, \\
R_{7}=\frac{z_{m}^{2}}{2 a_{m}}\left(a_{4} \sinh a_{m} z_{m}+a_{5} \cosh a_{m} z_{m}\right)-R_{70}, \\
R_{70}=\frac{z_{m}}{2 a_{m}^{2}}\left(a_{5} \sinh a_{m} z_{m}+a_{4} \cosh a_{m} z_{m}\right), \\
R_{8}=\frac{2 z_{m}\left(a_{7} \sinh \delta_{m} z_{m}+a_{6} \cosh \delta_{m} z_{m}\right)}{\delta_{m}^{2}-a_{m}^{2}}-R_{80},
\end{gathered}
$$




$$
\begin{aligned}
& R_{80}=\frac{4 \delta_{m}\left(a_{6} \sinh \delta_{m} z_{m}+a_{7} \cosh \delta_{m} z_{m}\right)}{\left(\delta_{m}^{2}-a_{m}^{2}\right)^{2}}, \\
& a_{20}=\hat{T}\left(a_{22} \cosh a_{m}+a_{23} \sinh a_{m}\right)-\Delta_{38}, \\
& a_{21}=\frac{a_{22} a_{m} \sinh a_{m}+a_{23} a_{m} \cosh a_{m}-\Delta_{39}}{a}, a_{22}=\frac{\Delta_{42}}{\Delta_{43}}, a_{23}=\frac{\Delta_{41}}{a_{m}}, \\
& \Delta_{38}=\hat{T}\left[\frac{1}{2 a_{m}}\left(a_{4} \sinh a_{m}+a_{5} \cosh a_{m}\right)-R_{380}+\Delta_{381}\right], \\
& \Delta_{380}=\frac{1}{2 a_{m}^{2}}\left(a_{5} \sinh a_{m}+a_{4} \cosh a_{m}\right), \\
& \Delta_{381}=\frac{4 \delta_{m}}{\left(\delta_{m}^{2}-a_{m}^{2}\right)^{2}}\left(a_{6} \sinh \delta_{m}+a_{7} \cosh \delta_{m}\right)+\Delta_{382}, \\
& \Delta_{382}=\frac{2}{\delta_{m}^{2}-a_{m}^{2}}\left(a_{7} \sinh \delta_{m}+a_{6} \cosh \delta_{m}\right), \\
& \Delta_{39}=-\left(\frac{a_{3}-a}{2 a^{3}}\right)+\frac{2 \delta_{m}}{\left(\delta_{m}^{2}-a_{m}^{2}\right)^{2}}\left(a_{6} \sinh \delta_{m}+a_{7} \cosh \delta_{m}\right)+\Delta_{390}+\Delta_{391} \text {, } \\
& \Delta_{390}=\frac{a_{4} \sinh a_{m}+a_{5} \cosh a_{m}}{2 a_{m}}+\frac{\left(a_{m}^{2}-1\right)\left(a_{5} \sinh a_{m}+a_{4} \cosh a_{m}\right)}{2 a_{m}^{2}}, \\
& \Delta_{391}=-\frac{2\left(\delta_{m}^{2}+a_{m}^{2}\right)}{\left(\delta_{m}^{2}-a_{m}^{2}\right)^{2}}\left(a_{7} \sinh \delta_{m}+a_{6} \cosh \delta_{m}\right), \\
& \Delta_{40}=\left(\frac{a_{2} a^{2}-a_{2}}{2 a^{2}}+\frac{1}{2 a}\right) \sinh a+\left(\frac{a^{2}-1}{2 a^{2}}+\frac{a_{2}}{2 a}\right) \cosh a+\Delta_{400}, \\
& \Delta_{400}=\left(\frac{3 a_{1}\left(a^{2}+1\right)+a a_{3}\left(2 a^{2}-3\right)}{6 a^{3}}\right) \sinh a+\Delta_{401}, \\
& \Delta_{401}=\left(\frac{3 a_{3}\left(a^{2}+1\right)+a a_{1}\left(2 a^{2}-3\right)}{6 a^{3}}\right) \cosh a, \\
& \Delta_{41}=-\frac{a_{4}}{2 a_{m}^{2}}-\frac{2 a_{6}\left(\delta_{m}^{2}+a_{m}^{2}\right)^{2}}{\left(\delta_{m}^{2}-a_{m}^{2}\right)^{2}}, \\
& \Delta_{42}=\Delta_{40}+\Delta_{39} \cosh a+a \Delta_{38} \sinh a-\Delta_{41} \cosh a \cosh a_{m}+\Delta_{420}, \\
& \Delta_{420}=-\frac{\Delta_{41}}{a_{m}}\left(\widehat{S}_{1} a \sinh a \sinh a_{m}\right), \Delta_{43}=\Delta_{25} .
\end{aligned}
$$

The thermal Marangoni number for this model obtained from $(47)^{1}$ and is found to be

$$
M_{2}=-\frac{\Lambda_{1}+\Lambda_{2}+\Lambda_{3}}{\Lambda_{5}}
$$


where

$$
\begin{gathered}
\Lambda_{5}=a^{2}\left[a_{20} \cosh a+a_{21} \sinh a-\Omega_{3}\right], \\
\Omega_{3}=\frac{1}{6 a^{3}}\left[R_{9}+R_{10}\right], \\
R_{9}=\left(3 a\left(a-a_{2}-a_{3}\right)+3 a_{1}+2 a^{2} a_{1}\right) \sinh a, \\
R_{10}=\left(3 a\left(a a_{2}-1-a_{1}\right)+3 a_{3}+2 a^{2} a_{3}\right) \cosh a .
\end{gathered}
$$

\subsection{Inverted Parabolic Temperature Profile}

We have

$$
h(z)=2(1-z) \text { and } h_{m}\left(z_{m}\right)=2\left(1-z_{m}\right)
$$

Substituting Equation (64) into the heat Equation (40) and Equation (44), we get $\theta$ and $\theta_{m}$ as

$$
\begin{gathered}
\theta(z)=A_{1}\left[a_{24} \cosh a z+a_{25} \sinh a z+\Psi(z)\right] \\
\theta_{m}\left(z_{m}\right)=A_{1}\left[a_{26} \cosh a_{m} z_{m}+a_{27} \sinh a_{m} z_{m}+\Psi_{m}\left(z_{m}\right)\right]
\end{gathered}
$$

where

$$
\begin{gathered}
\Psi(z)=-\left(R_{11}+R_{12}+R_{13}\right), \Psi_{m}\left(z_{m}\right)=-\left(R_{14}+R_{15}+R_{16}+R_{17}\right), \\
R_{11}=\left(\frac{z}{a}-\frac{z^{2}}{2 a}+\frac{a_{2} z}{2 a^{2}}\right) \sinh a z+\left(\frac{a_{2} z}{a}-\frac{a_{2} z^{2}}{2 a}+\frac{z}{2 a^{2}}\right) \cosh a z, \\
R_{12}=\left(\frac{z^{2}}{2 a}-\frac{z^{3}}{3 a}-\frac{z}{2 a^{3}}\right)\left(a_{1} \sinh a z+a_{3} \cosh a z\right), \\
R_{13}=\left(\frac{z^{2}-z}{2 a^{2}}\right)\left(a_{3} \sinh a z+a_{1} \cosh a z\right), \\
R_{14}=\left(\frac{z_{m}}{a_{m}}-\frac{z_{m}^{2}}{2 a_{m}}\right)\left(a_{4} \sinh a_{m} z_{m}+a_{5} \cosh a_{m} z_{m}\right), \\
R_{15}=\frac{z_{m}}{2 a_{m}^{2}}\left(a_{5} \sinh a_{m} z_{m}+a_{4} \cosh a_{m} z_{m}\right), \\
R_{16}=\frac{2\left(1-z_{m}\right)}{\delta_{m}^{2}-a_{m}^{2}}\left(a_{7} \sinh \delta_{m} z_{m}+a_{6} \cosh \delta_{m} z_{m}\right), \\
R_{17}=\frac{4 \delta_{m}}{\left(\delta_{m}^{2}-a_{m}^{2}\right)^{2}}\left(a_{6} \sinh \delta_{m} z_{m}+a_{7} \cosh \delta_{m} z_{m}\right), \\
a_{24}=\hat{T}\left(a_{26} \cosh a_{m}+a_{27} \sinh a_{m}\right)-\Delta_{44}, \\
a_{25}=\frac{1}{a}\left(a_{m} a_{27} \cosh a_{m}+a_{m} a_{26} \sinh a_{m}+\Delta_{45}\right), \\
a_{26}=\frac{\Delta_{48}}{\Delta_{49}}, a_{27}=\frac{\Delta_{47}}{a_{m}},
\end{gathered}
$$




$$
\begin{aligned}
& \Delta_{44}=\Delta_{440}+\frac{1}{2 a_{m}^{2}}\left(a_{5} \sinh a_{m}+a_{4} \cosh a_{m}\right), \\
& \Delta_{440}=\frac{1}{2 a_{m}}\left(a_{4} \sinh a_{m}+a_{5} \cosh a_{m}\right)+\Delta_{441}, \\
& \Delta_{441}=\frac{4 \delta_{m}}{\left(\delta_{m}^{2}-a_{m}^{2}\right)^{2}}\left(a_{6} \sinh \delta_{m}+a_{7} \cosh \delta_{m}\right), \\
& \Delta_{45}=\left(\frac{2 a^{2} a_{2}+a\left(1-a_{1}\right)-a_{3}}{2 a^{3}}\right)-\Delta_{450}-\Delta_{451}, \\
& \Delta_{450}=\frac{1}{2 a_{m}}\left(a_{4} \sinh a_{m}+a_{5} \cosh a_{m}\right), \\
& \Delta_{451}=\frac{1+a_{m}^{2}}{2 a_{m}^{2}}\left(a_{5} \sinh a_{m}+a_{4} \cosh a_{m}\right)+\Delta_{452}, \\
& \Delta_{452}=\frac{2\left(\delta_{m}^{2}+a_{m}^{2}\right)}{\left(\delta_{m}^{2}-a_{m}^{2}\right)^{2}}\left(a_{7} \sinh \delta_{m}+a_{6} \cosh \delta_{m}\right), \\
& \Delta_{46}=\frac{a_{2}+a_{2} a^{2}+a}{2 a^{2}} \sinh a+\frac{1+a^{2}+a a_{2}}{2 a^{2}} \cosh a-\Delta_{460}, \\
& \Delta_{460}=\left(\frac{a_{1}}{2 a^{3}}+\frac{a_{3}}{6}\right) \sinh a+\left(\frac{a_{3}}{2 a^{3}}+\frac{a_{1}}{6}\right) \cosh a, \\
& \Delta_{47}=\frac{a_{4}}{2 a_{m}^{2}}+\frac{a_{5}}{a_{m}}+2 a_{6} \frac{\delta_{m}^{2}+a_{m}^{2}}{\left(\delta_{m}^{2}-a_{m}^{2}\right)^{2}}+\frac{2 a_{7} \delta_{m}}{\delta_{m}^{2}-a_{m}^{2}}, \\
& \Delta_{48}=\Delta_{46}-\Delta_{49} \cosh a+a \Delta_{44} \sinh a-\Delta_{47} \cosh a \cosh a_{m}-\Delta_{480} \text {, } \\
& \Delta_{480}=\frac{\Delta_{47}}{a_{m}}\left(\hat{T} a \sinh a \sinh a_{m}\right), \Delta_{49}=\Delta_{25} .
\end{aligned}
$$

The thermal Marangoni number for this model obtained from $(47)^{1}$ and is found to be

$$
M_{3}=-\frac{\Lambda_{1}+\Lambda_{2}+\Lambda_{3}}{\Lambda_{6}}
$$

where

$$
\begin{gathered}
\Lambda_{6}=a^{2}\left[a_{24} \cosh a+a_{25} \sinh a-\Omega_{4}\right], \\
\Omega_{4}=\frac{1}{6 a^{3}}\left[\left(a^{2}-3\right)\left(a_{3} \cosh a+a_{1} \sinh a\right)\right]+R_{18}, \\
R_{18}=\frac{1}{2 a^{2}}\left[\left(a+a_{2}\right) \sinh a+\left(a a_{2}+1\right) \cosh a\right]
\end{gathered}
$$

\section{Results and Discussion}

The Thermal Marangoni numbers $M_{1}$ for linear, $M_{2}$ for parabolic and $M_{3}$ for inverted parabolic temperature profiles are obtained. The constraints are drawn 
against the depth ratio $\hat{d}$. The dimensionless fixed values are $\hat{T}=1.0, \hat{S}=1.0$, $a=1.0, \quad \beta=0.03, \quad M_{s 1}=10, \quad M_{s 2}=1, \tau_{1}=\tau_{2}=\tau_{m 1}=\tau_{m 2}=\widehat{S_{1}}=\widehat{S_{2}}=0.25$ and $\hat{\mu}=2.5$.

The effects of the parameters $a, \beta, \hat{\mu}, \tau_{1}, \tau_{m 1}, \widehat{S}_{1}, M_{s 1}$ and $M_{s 2}$ on all the three thermal Marangoni numbers are depicted in Figures 1 to 8 . The main observation that the thermal Marangoni numbers of all the three profiles, the inverted

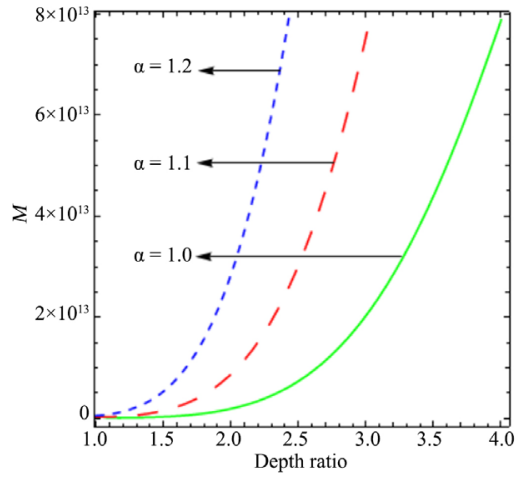

(a)

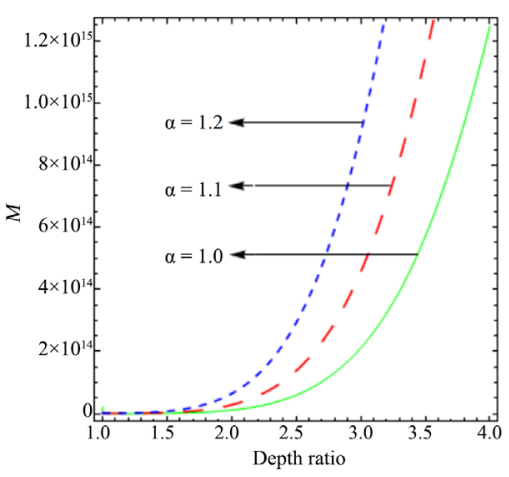

(b)

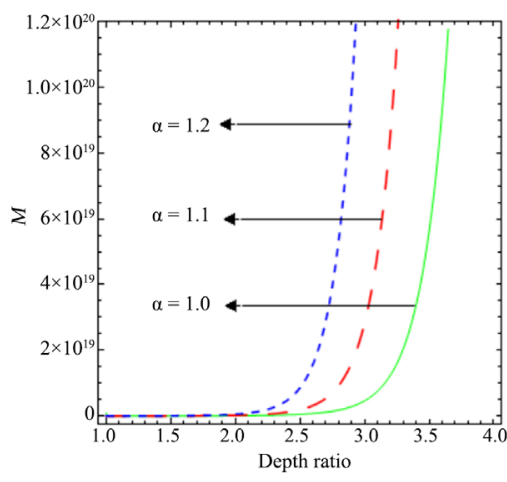

(c)

Figure 1. The effects of horizontal wave number $a$.

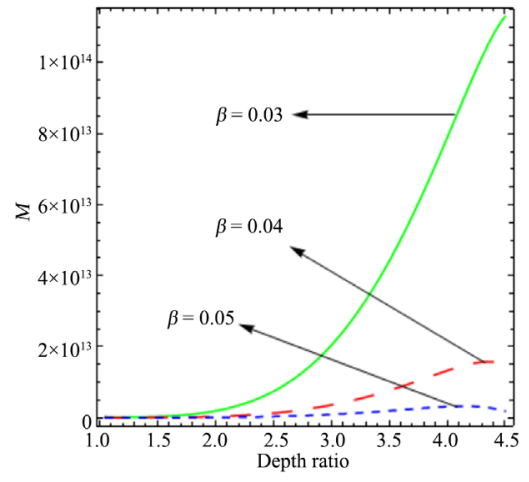

(a)

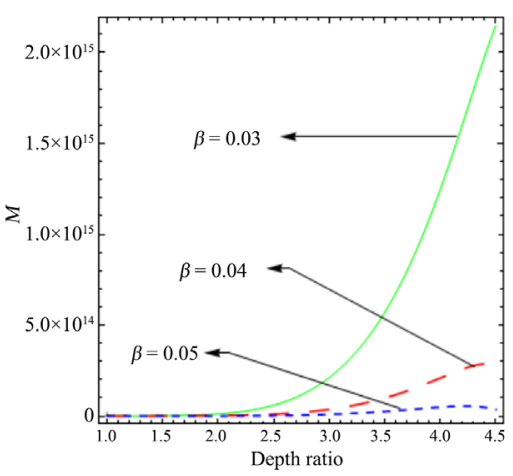

(b)

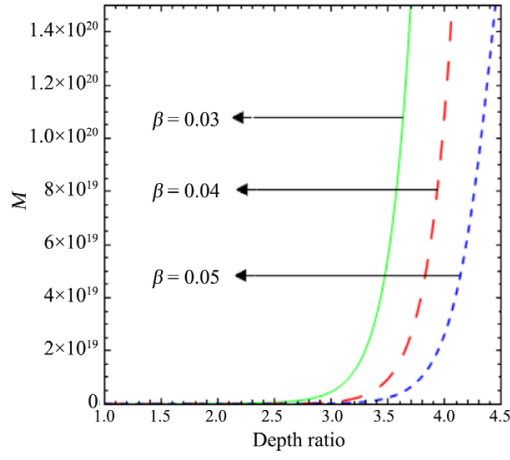

(c)

Figure 2. The effects of porous parameter $\beta$.

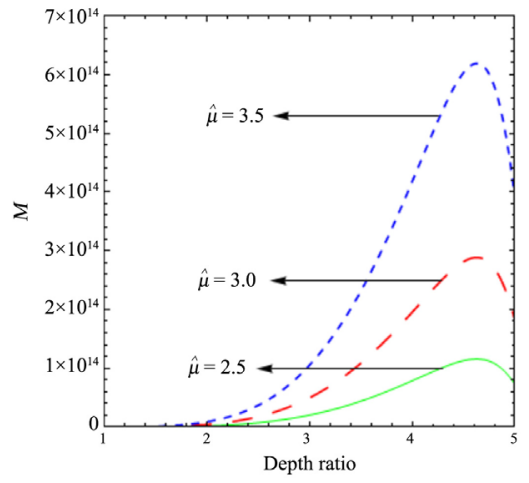

(a)

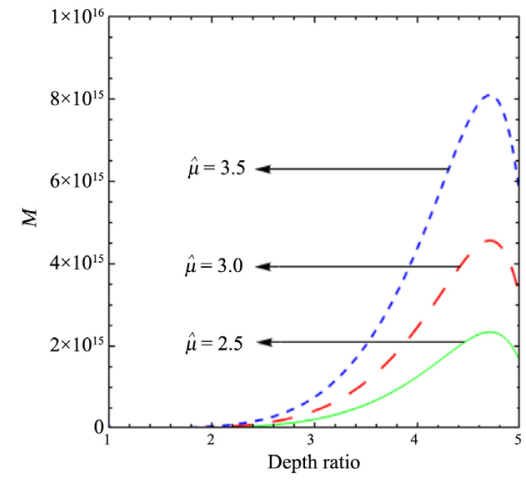

(b)

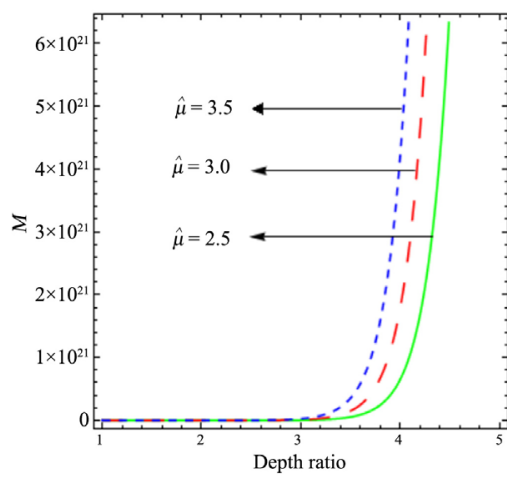

(c)

Figure 3 . The effects of viscosity ratio $\hat{\mu}$. 


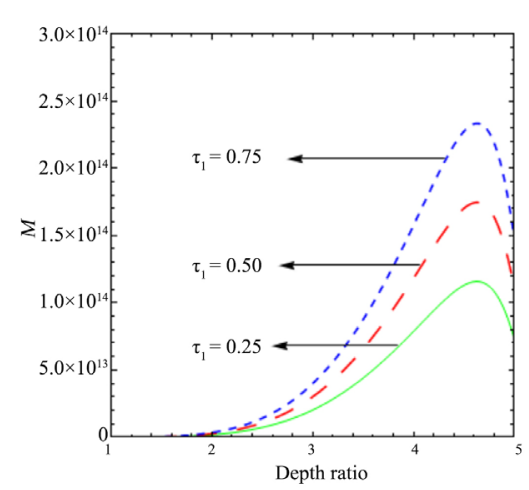

(a)

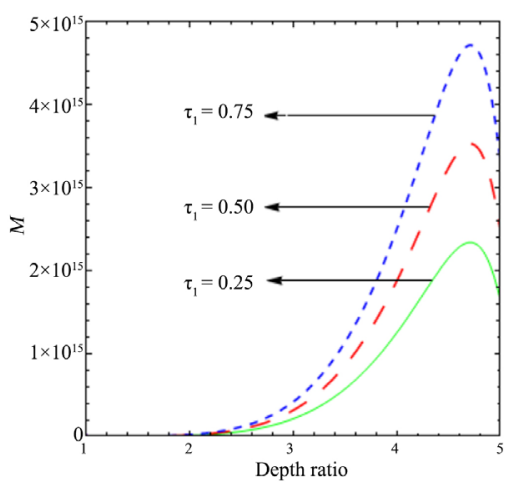

(b)

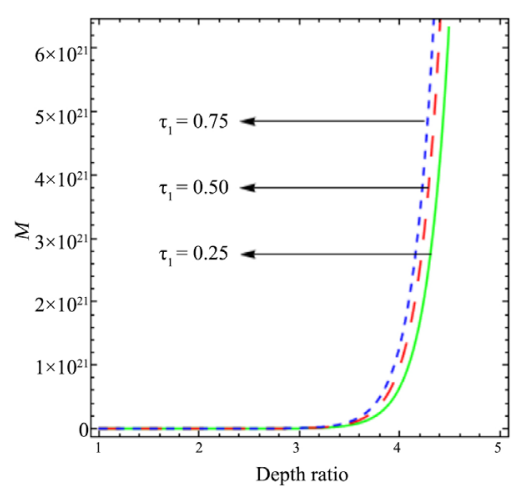

(c)

Figure 4. The effects of $\tau_{1}$.

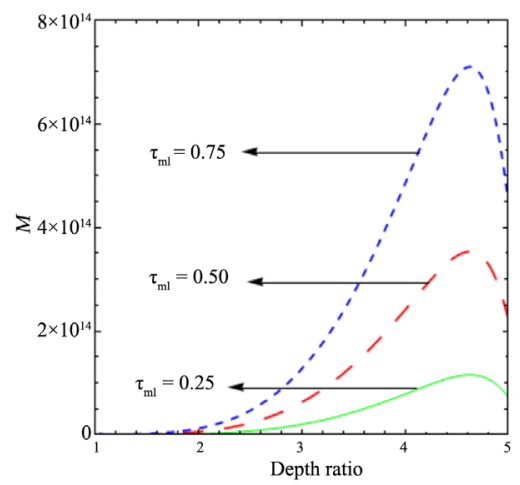

(a)

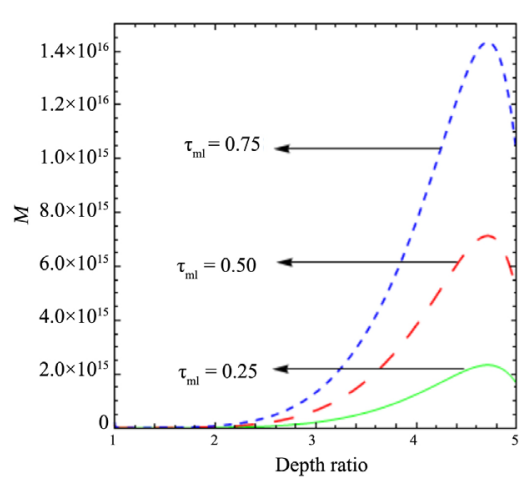

(b)

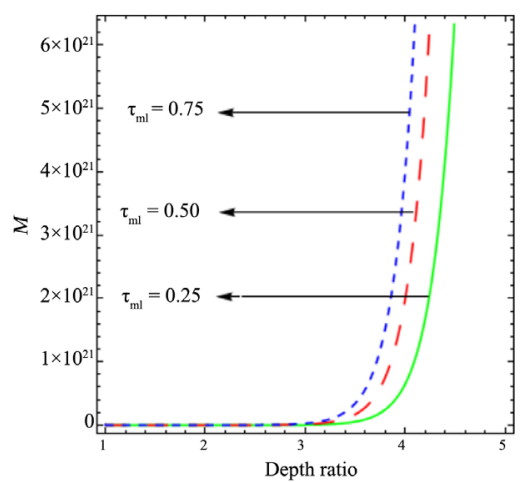

(c)

Figure 5. The effects of $\tau_{m 1}$.

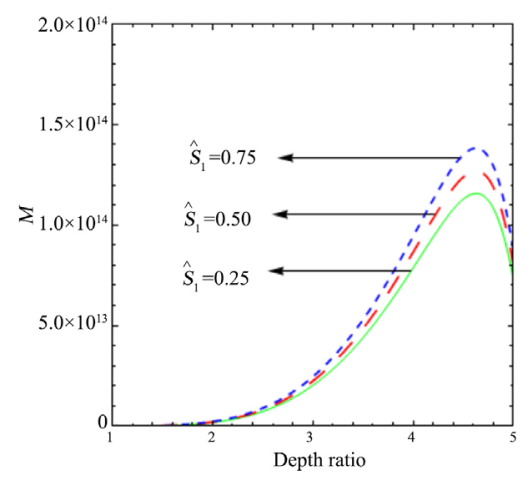

(a)

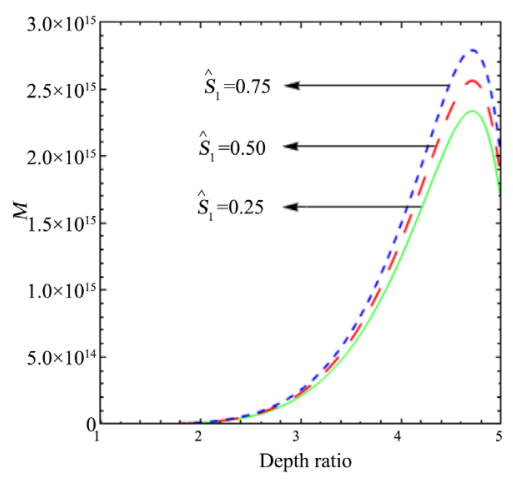

(b)

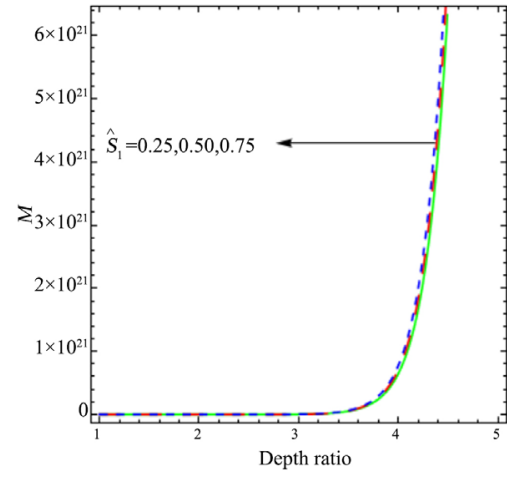

(c)

Figure 6. The effects of $\widehat{S_{1}}$.

parabolic profile is the most stable one and the linear profile is the most unstable one as the thermal Marangoni numbers are highest and lowest respectively, for a given set of fixed values of parameters, specially for porous layer dominant systems. For fluid dominant system, there is no much change in the thermal Marangoni numbers for all the profiles.

The variations of $a$, horizontal wave number on the thermal Marangoni 


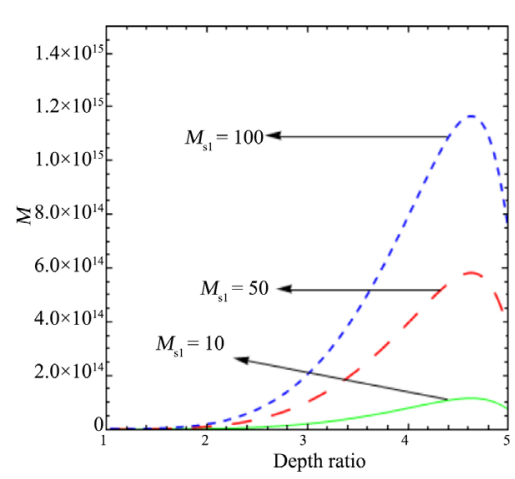

(a)

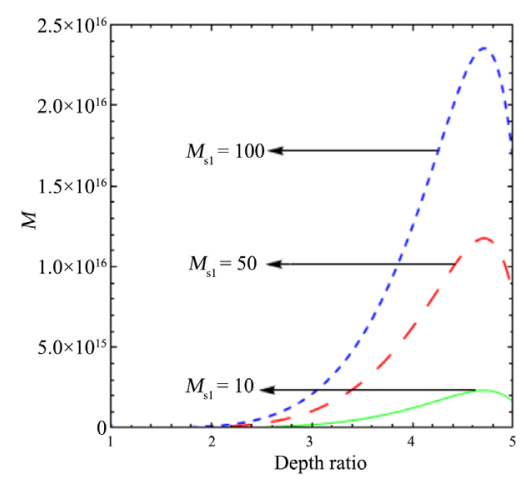

(b)

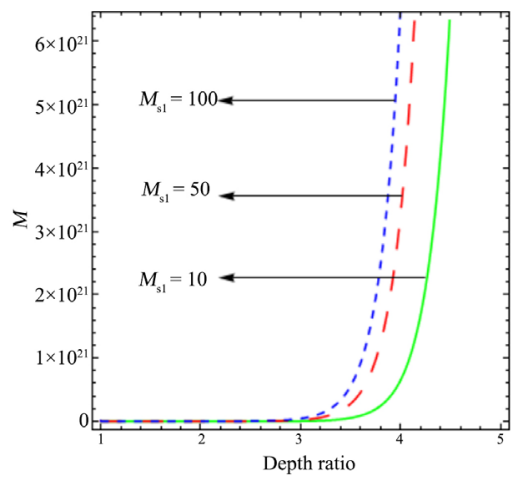

(c)

Figure 7. The effects of solute1 Marangoni number $M_{s 1}$.

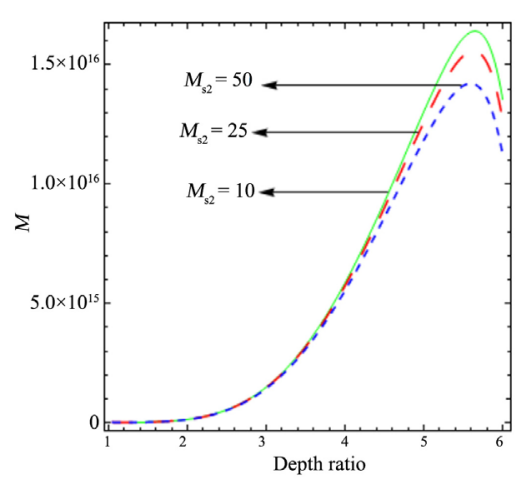

(a)

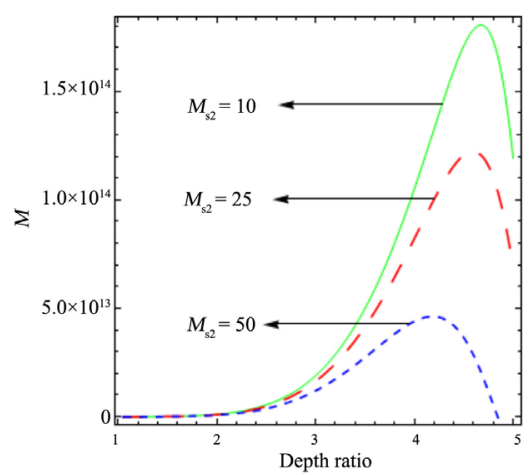

(b)

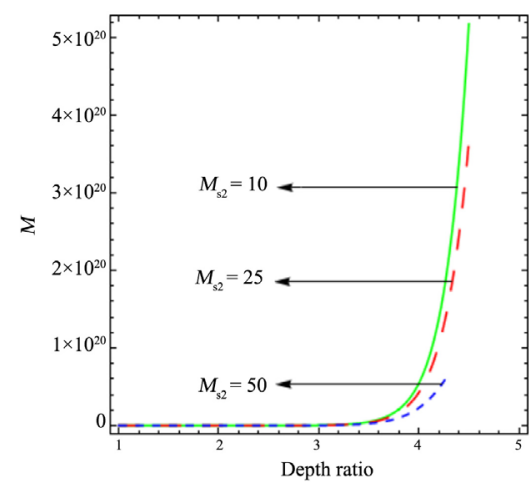

(c)

Figure 8. The effects of solute2 Marangoni number $M_{s 2}$.

numbers $M_{1}, M_{2}$ and $M_{3}$ are respectively shown in Figures 1(a)-(c) for $a=1.0,1.1$ and 1.2. We observed that the thermal Marangoni number for the inverted parabolic profile is larger than those for the linear and parabolic profiles. For all the profiles, it is evident from the graph that an increase in the value of $a$, the thermal Marangoni number increases and its effect is to stabilize the system.

The variations of the porous parameter $\beta$ on the three thermal Marangoni numbers are depicted Figures 2(a)-(c). The curves for $\beta=0.03,0.04,0.05$. Increase in the value of $\beta$, i.e., increasing the permeability, the thermal Marangoni numbers decrease for all the three profiles. Hence the surface tension driven triple diffusive convection occurs earlier on increasing the porous parameter, which is physically reasonable, as there is more way for the fluid to move. So, the system is destabilized.

Figures 3(a)-(c) show the variations of viscosity ratio $\hat{\mu}$ for the values $\hat{\mu}=2.5,3.0,3.5$. Increase in the value of $\hat{\mu}$, the values of the thermal Marangoni numbers $M_{1}, M_{2}$ and $M_{3}$ increases. So, the increase in the values of viscosity ratio is to stabilize the system and hence the surface tension driven triple diffusive convection is delayed.

Figures 4(a)-(c) display the effects of $\tau_{1}$ is the ratio of salutel diffusivity to thermal diffusivity fluid in fluid layer for $M_{1}, M_{2}$ and $M_{3}$ respectively for the 
values $\tau_{1}=0.25,0.50,0.75$. For all the three profiles, there is a increase in the values of the thermal Marangoni numbers. Increasing the value of $\tau_{1}$ the surface tension driven triple diffusive convection becomes slow and hence the system can be stabilized.

Figures 5(a)-(c) display the variations of the value of $\tau_{m 1}$ is the ratio of salute1 diffusivity to thermal diffusivity of the porous layer for the values $\tau_{m 1}=0.25,0.50,0.75$. Increasing this ratio, the thermal Marangoni numbers increase for all the three profiles. So, the surface tension driven triple diffusive convection becomes slow and hence the system can be stabilized.

Figures 6(a)-(c) show the effects of ratio of solutel diffusivity of the fluid in the fluid layer to that of porous layer $\widehat{S_{1}}=0.25,0.50,0.75$. Increasing this ratio, for all the three profiles, there is a small increase in $M_{1}, M_{2}$ and $M_{3}$ so, the surface tension driven triple diffusive convection becomes slow and hence the system can be stabilized.

Figures 7(a)-(c) show the effects of the $M_{s 1}$ is the solute1 Maran-goni number for $M_{s 1}=10,50,100$. By increasing the values of Solute1 Marangoni numbers, the thermal Marangoni numbers increase for all the three temperature profiles. So, the surface tension driven triple diffusive convection can be delayed by increasing solute Marangoni number, hence the system can be stabilized.

Figures 8(a)-(c) illustrate the effects of the $M_{s 2}$ is the solute2 Marangoni number for $M_{s 2}=10,25,50$. By increasing the values of Solute2 Marangoni numbers, the thermal Marangoni numbers decrease for all the three temperature profiles. So, the surface tension driven triple diffusive convection can be preponed by increasing solute Marangoni number, hence the system can be destabilized.

\section{Conclusions}

1) The inverted parabolic temperature profile is the most suitable for the situations demanding the control of Marangoni convection, whereas the linear and parabolic profile is suitable for the situations where the convection is needed.

2) By increasing the values of $a, \hat{\mu}, \tau_{1}, \tau_{m 1}, \widehat{S_{1}}, M_{s 1}$ and by decreasing the values of $\beta$ and $M_{\mathrm{s} 2}$ the surface tension driven triple diffusive convection in a composite layer under microgravity condition can be delayed and hence the system can be stabilized.

3) In the manufacture of pure crystal growth, our work can be useful. The people who are manufacturing crystals can refer this paper. This can give them an initial insight into the effects of parameters in the multicomponent crystal growth problems.

\section{Acknowledgements}

We express our gratitude to Prof. N. Rudraiah and Prof. I. S. Shivakumara, UGC-CAS in Fluid mechanics, Bangalore University, Bengaluru, for their help during the formulation of the problem. The author Manjunatha. $\mathrm{N}$, express his sincere thanks to the management of REVA University, Bengaluru, for their 
encouragement.

\section{Conflicts of Interest}

The authors declare no conflicts of interest regarding the publication of this paper.

\section{References}

[1] Griffiths, R.W. (1979) The Influence of Third Diffusing Component upon the Onset of Convection. Journal of Fluid Mechanics, 92, 659-670. https://doi.org/10.1017/S0022112079000811

[2] Griffiths, R.W. (1979) A Note on the Formation of Salt-Finger and Diffusive Interfaces in Three Component Systems. International Journal of Heat and Mass Transfer, 22, 1687-1693. https://doi.org/10.1016/0017-9310(79)90085-1

[3] Rudraiah, N. and Vortmeyer, D. (1982) The Influence of Permeability and of a Third Diffusing Component upon the Onset of Convection in a Porous Medium. International Journal of Heat and Mass Transfer, 25, 457-464. https://doi.org/10.1016/0017-9310(82)90049-7

[4] Shivakumara, I.S. (1985) Convection of Two and Three Component Systems in a Horizontal Layer. PhD Thesis, Bangalore University, Bangalore.

[5] Poulikakos, D. (1985) The Effect of Third Diffusing Component on the Onset of Convection in a Horizontal Porous Layer. Physics of Fluids, 28, 3172. https://doi.org/10.1063/1.865359

[6] Pearlstein, A.J., Haris, R.M. and Terrones, G. (1989) The Onset of Convective Instability in a Triply Diffusive Fluid Layer. Journal of Fluid Mechanics, 202, 443-465. https://doi.org/10.1017/S0022112089001242

[7] Lopez, A.R., Louis, A.R. and Pearlstein, A.J. (1990) Effect of Rigid Boundaries on the Onset of Convective Instability in Triply Diffusive Fluid Layer. Physics of Fluids A, 2, 897. https://doi.org/10.1063/1.857650

[8] Chand, S. (2013) Linear Stability of Triple-Diffusive Convection in Micropolar Ferromagnetic Fluid Saturating Porous Medium. Applied Mathematics and Mechanics, 34, 309-326. https://doi.org/10.1007/s10483-013-1672-9

[9] Chand, S. (2013) Triple-Diffusive Convection in a Micropolar Ferrofluid in the Presence of Rotation. International Journal of Applied Mechanics and Engineering, 18, 307-327. https://doi.org/10.2478/ijame-2013-0018

[10] Shivakumara, I.S. and Naveen Kumar, S.B. (2014) Linear and Weakly Nonlinear Triple Diffusive Convection in a Couple Stress Fluid Layer. International Journal of Heat and Mass Transfer, 68, 542-553. https://doi.org/10.1016/j.ijheatmasstransfer.2013.09.051

[11] Kango, S.K., Sharma, S. and Chada, K. (2016) Triple-Diffusive Convection in a Micropolar Rotating Ferrofluid. International Journal of Technology, 6, 123-132. https://doi.org/10.5958/2231-3915.2016.00020.1

[12] Kumar, V. and Awasthi, M.K. (2016) Onset of Triple-Diffusive Convection in a Nanofluid Layer. Journal of Nanofluids, 5, 284-291.

https://doi.org/10.1166/jon.2016.1217

[13] Tarannum, S. and Pranesh, S. (2016) Triple Diffusive Convection in Oldroyd-B Liquid. IOSR Journal of Mathematics, 12, 7-13. https://doi.org/10.9790/5728-1204010713 
[14] Tarannum, S. and Pranesh, S. (2017) Heat and Mass Transfer of Triple Diffusive Convection in a Rotating Couple Stress Liquid Using Ginzburg-Landau Model. International Journal of Mechanical and Mechatronics Engineering, 11, 583-588.

[15] Chand, S. (2012) Effect of Rotation on Triple-Diffusive Convection in a Magnetized Ferrofluid with Internal Angular Momentum Saturating a Porous Medium. Applied Mathematical Sciences, 6, 3245-3258.

[16] Rionero, S. (2013) Triple Diffusive Convection in Porous Media. Acta Mechanica, 224, 447-458. https://doi.org/10.1007/s00707-012-0749-2

[17] Kango, S.K., Rana, G.C. and Chand, R. (2013) Triple-Diffusive Convection in Walters (Model b) Fluid with Varying Gravity Field Saturating a Porous Medium. Studia Geotechnica et Mechanica, 35, 45-56. https://doi.org/10.2478/sgem-2013-0029

[18] Khan, Z.H., Khan, W.A. and Pop, I. (2013) Triple Diffusive Free Convection along a Horizontal Plate in Porous Media Saturated by a Nanofluid with Convective Boundary Condition. International Journal of Heat and Mass Transfer, 66, 603-612. https://doi.org/10.1016/j.ijheatmasstransfer.2013.07.074

[19] Zhao, M.L., Wang, S.W. and Zhang, Q.Y. (2014) Onset of Triply Diffusive Convection in a Maxwell Fluid Saturated Porous Layer. Applied Mathematical Modelling, 38, 2345-2352. https://doi.org/10.1016/j.apm.2013.10.053

[20] Sharma, P.K., Malik, H., Kumar, V. and Kumar, P. (2014) Triply-Diffusive Magneto Convection in Viscoelastic Fluid through Porous Medium. International Transactions in Applied Sciences, 6, 495-510.

[21] Prakash, J., Bala, R. and Vaid, K. (2015) On the Characterization of Magnetohydrodynamic Triply Diffusive Convection. Journal of Magnetism and Magnetic Materials, 377, 378-385. https://doi.org/10.1016/j.jmmm.2014.10.102

[22] Prakash, J., Singh, V. and Manan, S. (2017) On the Limitations of Linear Growth Rates in Triply Diffusive Convection in Porous Medium. Journal of the Association of Arab Universities for Basic and Applied Sciences, 22, 91-97. https://doi.org/10.1016/j.jaubas.2015.12.002

[23] Rana, G.C., Chand, R., Sharma, V. and Sharda, A. (2016) On the Onset of Triple-Diffusive Convection in a Layer of Nano Fluid. Journal of Computational Applied Mechanics, 47, 67-77.

[24] Goyal, M., Goyal, R. and Bhargava, R. (2017) Fem Simulation of Triple Diffusive Natural Convection along Inclined Plate in Porous Medium: Prescribed Surface Heat. Solute and Nanoparticles Flux, 22, 883-900. https://doi.org/10.1515/ijame-2017-0057

[25] Patil, P.M., Roy, M., Roy, S. and Momoniat, E. (2018) Triple Diffusive Mixed Convection along a Vertically Moving Surface. International Journal of Heat and Mass Transfer, 117, 287-295. https://doi.org/10.1016/j.ijheatmasstransfer.2017.09.106

[26] Awasthi, M.K., Kumar, V. and Patel, R.K. (2018) Onset of Triply Diffusive Convection in a Maxwell Fluid Saturated Porous Layer with Internal Heat Source. Ain Shams Engineering Journal, 9, 1591-1600. https://doi.org/10.1016/j.asej.2016.11.012

[27] Raghunatha, K.R., Shivakumara, I.S. and Shankar, B.M. (2018) Weakly Nonlinear Stability Analysis of Triple Diffusive Convection in a Maxwell Fluid Saturated Porous Layer. Applied Mathematics and Mechanics, 39, 153-168.

https://doi.org/10.1007/s10483-018-2298-6

[28] Sumithra, R. (2012) Exact Solution of Triple Diffusive Marangoni Convection in a Composite Layer. International Journal of Engineering Research \& Technology, 1, 
1-13.

[29] Manjunatha, N. and Sumithra, R. (2018) Effects of Non-Uniform Temperature Gradients on Double Diffusive Marangoni Convection in a Two Layer System. International Journal of Pure and Applied Mathematics, 118, 203-220.

[30] Manjunatha, N. and Sumithra, R. (2018) Effects of Non-Uniform Temperature Gradients on Surface Tension Driven Two Component Magnetoconvection in a Porous-Fluid System. ARPN Journal of Engineering and Applied Sciences, 13, 429-441.

[31] Chen, F. and Chen, C.F. (1998) Onset of Finger Convection in a Horizontal Porous Layer Underlying a Fluid Layer. Journal of Heat Transfer, 110, 403. https://doi.org/10.1115/1.3250499

[32] Nield, D.A. (1977) Onset of Convection in a Fluid Layer Overlying a Layer of a Porous Medium. Journal of Fluid Mechanics, 81, 513-522. https://doi.org/10.1017/S0022112077002195

[33] Sparrow, E.W., Goldstein, R.J. and Jonson, V.K. (1964) Thermal Instability in a Horizontal Fluid Layer: Effect of Boundary Conditions and Nonlinear Temperature Profile. Journal of Fluid Mechanics, 18, 513-528.

https://doi.org/10.1017/S0022112064000386 